\title{
Throughput Maximization and Fairness Assurance in Data and Energy Integrated Communication Networks
}

\author{
Kesi Lv, Jie Hu, Member, IEEE, Qin Yu, and Kun Yang, Senior Member, IEEE
}

\begin{abstract}
A typical data and energy integrated network (DEIN) conceives a conventional base station (BS), which is capable of simultaneously transmitting the data and energy to user equipments (UEs) during the downlink (DL) transmissions by invoking the time-division-multiple-access (TDMA) protocol in the medium access control (MAC) layer. Several UEs operating in this DEIN are capable of harvesting the energy from the DL transmissions by adopting the power splitting (PS) technique and they are also capable of exploiting the harvested energy for powering their uplink (UL) data transmissions by invoking the TDMA protocol in the MAC layer. Both of the UL sumthroughput and the UL fair-throughput of the DEIN is maximised by deciding the duration of each time-slot during the DL/UL transmissions and by determining the optimal PS factor for each UE. Both of these optimization problems are finally solved by the classic method of Lagrange multipliers in close-form. An interesting observation shows that supporting low-throughput data services during the DL transmissions does not degrade the wireless energy transfer and hence does not reduce the throughput of the UL transmissions.
\end{abstract}

Index Terms-Internet of Things, Data and Energy Integrated Communication Network (DEIN), Sum-Throughput/FairThroughput Maximisation

\section{INTRODUCTION}

Our cities now are in the process of transiting towards more smart, more automatic and more responsive societies, which requires the integration of the modern communication and information technology and the Internet of Things (IoT) [1]. The assets of smart cities contains smart transportation systems [2], smart grids [3], smart hospitals [4], smart factories [5] and etc. All these realisations require the universal connectivity of humans and machines. As foreseen by the industry, we will see more than 200000 IoT devices deployed in a square kilometre.

Conventionally, the energy supplies of user equipments ${ }^{1}$ (UEs) in wireless communication networks come from either

Kesi Lv, Jie Hu and Qin Yu are with the School of Communication and Information Engineering, University of Electronic Science and Technology of China, Chengdu, 611731, China, email: kslv@ std.uestc.edu.cn, \{hujie, yuqin\}@uestc.edu.cn. Dr. Jie Hu is the corresponding author of this article.

Kun Yang are with the the School of Computer Science \& Electronic Engineering, University of Essex, Essex, CO4 3SQ, UK, email: kunyang@essex.ac.uk

The financial support of University of Electronic Science and Technology of China, No. ZYGX2016KYQD103, and that of National Natural Science Foundation of China (NSFC), Grant No. 61601097, are gratefully acknowledged.

Copyright (c) 2012 IEEE. Personal use of this material is permitted. However, permission to use this material for any other purposes must be obtained from the IEEE by sending a request to pubs-permissions@ieee.org.

${ }^{1}$ The advent of IoT redefines the concept of user equipments, which now includes both hand-held devices and machine-type devices. batteries embedded or the power grid connected. Howerver, these two energy sources have obvious limitations. The limited energy stored in the batteries restricts the life time of UEs, while the wire connected to the power grid restricts UEs' movement. Furthermore, massive IoT devices are deployed in walls or under roads or in other untouchable places. It is difficult to regularly replace their batteries, which limit their life time. Embedding the function of energy harvesting (EH) into UEs and seeking energy from the renewable sources, such as sunlight [6] and wind [7], are capable of satisfying UEs' increasing energy demand [8]. However, energy arrivals from the renewable sources are stochastic processes, which hinders its efficient usage in supporting the communication functions of the UEs

Transferring energy by RF signals is more reliable and controllable than renewable energy sources . Zungeru and $e t$ $a l$. has demonstrated the availability of harvesting energy from the surrounding RF signals [9]. Varsheney has provided an information theoretical anaysis for revealing the performance limit of simultaneous wireless information and power transfer (SWIPT) [10]. In order to process contaminated RF signals for the information reception as well as to convert RF signals into direct-current (DC) for the energy harvesting, the spatial splitting (SS) [11], the power splitting (PS) [12] and the time switching (TS) [13] techniques have been invoked for the SWIPT. Many efforts then have been contributed to this prosperous subject [14]-[17]. However, most of them are merely based on the frequency-division-multiple-access (FDMA) protocol, while assuming symmetric duration of the UEs' donwlink (DL) and uplink (UL) transmissions. Their optimization formulation is inapplicable, when the time-divisionmultiple-access (TDMA) protocol is adopted in the MAC layer for supporting the information and energy transfer in the multiuser scenario, since their methodologies failed to optimize the durations of both the DL and the UL transmissions. Furthermore, wireless powered communication networks (WPCNs) relying on the TDMA protocol have been investigated in [18] [20]. In WPCNs, the protocol of "harvest-then-transfer" is conceived [21]. As a result, UEs may harvest energy from the base station (BS) during their DL transmissions, then the energy harvested by the UEs is exploited for supporting their UL transmissions. However, in WPCNs, the DL transmissions are dedicated to the wireless energy transfers. The simultaneous energy and data transfer has been largely ignored. Against this background, our novel contributions are summarised as follows: 


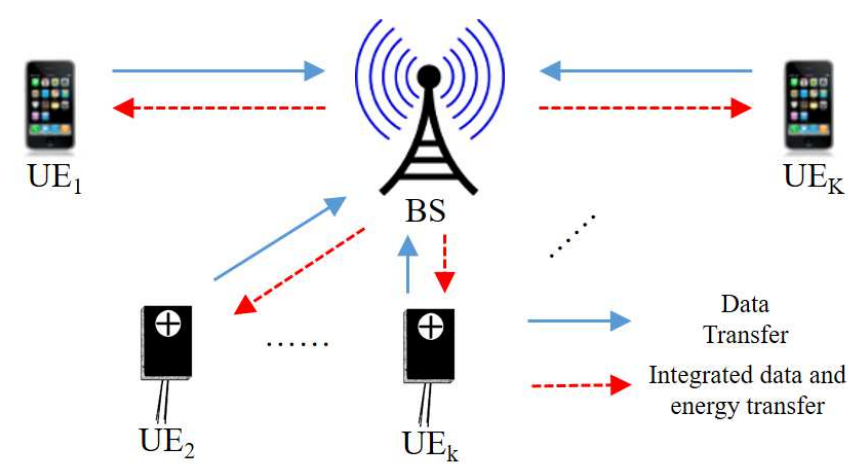

Fig. 1: The DL and UL transmissions of the DEIN.

- A novel data and energy integrated communication network (DEIN) is systematically established. In this DEIN, the BS simultaneously transfers both of the information and the energy to the UEs by obeying the TDMA protocol in the MAC layer during the DL transmissions. Then the UEs initiate their UL data transmissions by exploiting the energy harvested during the DL transmission stage.

- Relying on the tool of the convex optimization and the classic method of the Lagrange multipliers, the sumthroughput maximization problem for the UL transmissions is solved by jointly optimizing the allocation of the time slots for both of the DL and UL transmissions and the diverse PS factors for all the UEs.

- In order to further ensure the fairness among the UEs in the DEIN, the fair-throughput, which is defined as the minimum throughput among all the UEs' UL transmissions, is also maximized by optimizing the allocation of the time slots for both of the DL and UL transmissions and the diverse PS factors for all the UEs.

The rest of this paper is organised as follows. Our DEIN model is introduced in Section II, followed by the maximisation of the sum-throughput as well as the fair-throughput of the UL transmissions in Sections III and IV, respectively. Numerical results are provided in Section V. Finally, we conclude our paper in Section VI

\section{SySTEM MOdeL}

We consider a typical DEIN, as portrayed in Fig.1, for the sake of remotely charging the UEs without violating their communication demands. The DEIN consists of a single BS as well as $K$ UEs, which are denoted by the set $\left\{U_{i} \mid i=1, \cdots, K\right\}$. The BS and the UEs all conceive a single antenna and they operate on the same spectral band, which indicates that all the spatial resources and frequency resources are multiplexed by the UEs. Moreover, the UEs in the DEIN are equipped with super capacitors [22]. Super capacitors may ideally store the energy that is extracted from RF signals without any energy loss. However, super capacitors suffer from low energy storage capacity. As a result, the UEs have to harvest energy from the DL transmissions of the BS and store the energy in the super capacitors, while simultaneously extracting their requested data information from the same RF signals. The

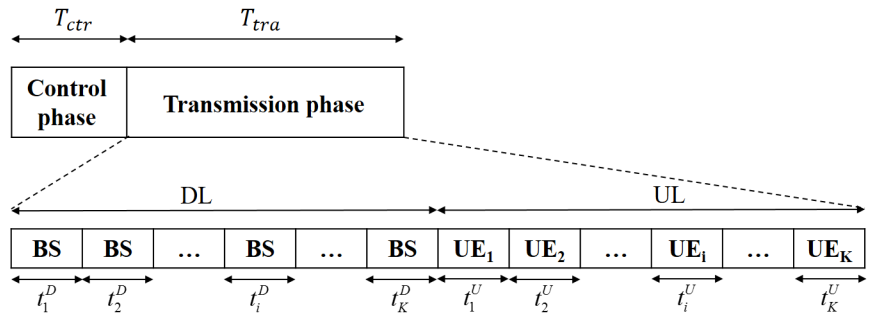

(a) The structure of a single operating cycle

\begin{tabular}{|c|c|c|c|c|c|c|c|c|c|c|}
\hline \multicolumn{6}{|c|}{$\mathrm{DL}$} & \multicolumn{5}{|c|}{ UL } \\
\hline EH & \begin{tabular}{|l|} 
EH \\
\end{tabular} & $\ldots$ & EH\&ID & \begin{tabular}{l|l}
$\ldots .$. & EH
\end{tabular} & St & St & $\ldots$ & IT & $\ldots$ & St \\
\hline
\end{tabular}

(b) $U_{i}$ 's operating mode during a specific cycle.

Fig. 2: Slotted DL/UL transmissions in the DEIN.

energy stored in super capacitors is then depleted for powering the UEs' UL transmissions. We further assume that the channel state information (CSI) is known by the BS.

\section{A. Structure of the TDMA aided operating cycle}

In the DEIN studied, the UEs are fully powered by the energy gleaned from the DL RF signals. As a result, their transmit power of the UL transmissions is very low. The timedivision-multiple-access (TDMA) protocol is then adopted for avoiding hostile interference and collision, when multiple UEs upload their data to the BS. Furthermore, adopting the TDMA protocol for the DL transmissions is capable of orthogonally transmitting data to the requesters. The UEs may also flexibly switch between the ID and EH operations in the time domain during the DL transmissions of the BS.

The structure of an operating cycle having a length of $T$ is depicted in Fig.2. An intact operating cycle consists of two phases, namely the control phase having a duration of $T_{c t r}$ and the transmission phase having a duration of $T_{t r a}$. During the control phase, the following tasks have to be completed by exchanging control signalling between the BS and the UEs:

- Channel Estimation: the channel state information (CSI) can be acquired by the BS via the forward-link training together with the reverse-link feedback [23]. The channel states are assumed unchanged during a single operating cycle but they vary from one operating cycle to another.

- Resource Allocation: given the CSI, the BS executes the time slot allocation for both the UEs' DL and UL transmissions and the BS also determines the signal splitting strategies at the UEs for simultaneous data and energy reception. The BS then notifies the UEs about the time slot allocation scheme and the signal splitting strategies.

- Synchronisation: since all the UEs are distributed in the coverage of the BS, they may readily be synchronised together by invoking the time-stamp-based synchronization approach [24]. The BS may broadcast its locally recorded clock information to all the UEs during the control phase. Once the clock information is successfully received, the UEs may adjust their local clock in order to complete their synchronisation process. 
The transmission phase of a single operating cycle is divided into a range of DL time slots denoted by the set of $\mathbf{t}^{D}=$ $\left\{t_{i}^{D} \mid i=1,2 \cdots, K\right\}$ and a range of UL time slots denoted by the set of $\mathbf{t}^{U}=\left\{t_{i}^{U} \mid i=1,2 \cdots, K\right\}$. Hence, we have the following inequality, which is expressed as

$$
\sum_{i=1}^{K}\left(t_{i}^{D}+t_{i}^{U}\right) \leq T_{\text {tra }} .
$$

The BS sends information to the requester $U_{i}$ during the DL time slot $t_{i}^{D}$, while $U_{i}$ sends its own data to the BS during the UL time slot $t_{i}^{U}$. Fig.2(b) presents how $U_{i}$ operates during a single operating cycle $T$. When the BS sends the data to another requester $U_{j}$ during a specific DL time slot $t_{j}^{D}, U_{i}$ $(i \neq j)$ may detect the RF signal emitted by the BS due to the broadcast nature of the wireless channel. Hence, $U_{i}$ is capable of harvesting the energy from the RF signal dedicated to its peer $U_{j}$. Hence $U_{i}$ operates in the EH mode during the current DL time slot $t_{j}^{D}$. During its own dedicated DL time slot $t_{i}^{D}, U_{i}$ adopts the PS technique for splitting the power $P_{\text {recv }, i}$ of its dedicated RF signal into two portions. The power of $\rho_{i} P_{\text {recv }, i}$ is relied upon for the energy harvesting, while the rest is for the information decoding (ID), where the parameter $\rho_{i}$ is regarded as the PS factor of $U_{i}$. As a result, $U_{i}$ simultaneously operates in the EH mode and the ID mode during $t_{i}^{D}$. The PS factor $\rho_{i}$ can be adjusted by $U_{i}$ in order to fulfil different energy and data requirements. For the UL transmission, since only a single UE is allowed to transfer its data during a specific time slot, $U_{i}$ solely operates in the information transfer (IT) mode during its assigned UL time slot $t_{i}^{U}$. By contrast, $U_{i}$ operates on the standby (St) mode during other UL time slots $\left\{t_{j}^{U} \mid j \neq i\right\}$ in order to avoid any transmission collision when the corresponding UE $U_{j}$ operates in the IT mode.

\section{B. Channel model}

The DL channel from the BS to $U_{i}$ and the corresponding reversed UL channel are denotd by the complex random variables $\tilde{h}_{i}$ and $\tilde{g}_{i}$, respectively, while their power gains are denoted by $h_{i}=\left|\tilde{h}_{i}\right|^{2}$ and $g_{i}=\left|\tilde{g}_{i}\right|^{2}$. For simplicity, we assume a symmetric channel between the BS and $U_{i}$, which indicates $h_{i}=g_{i}$. The set of channel power gains is denoted as $\mathbf{h}=\left\{h_{i} \mid i=1, \cdots, K\right\}$. Furthermore, the uncorrelated block fading channel models are conceived, which indicats that the power gain of the channel remain unchanged during a single operating cycle $T$. The channel noise power is denoted by $\sigma_{c, i}^{2}$, while the noise power of the ID is denoted by $\sigma_{I D, i}^{2}$. Compare to $\sigma_{I D, i}^{2}$, the channel noise power $\sigma_{c, i}^{2}$ is negligibly small and hence it has little influence on both of the practical ID and the EH [12]. As a result, the channel noise power $\sigma_{c, i}^{2}$ can be reasonably ignored in any of the formulations below. Furthermore, the noise power of ID is assumed to be identical for every UE as well as the BS. For simplicity, we let $\sigma^{2}=\sigma_{I D, i}^{2}$ to denote noise power of the ID in the following problem formulation.

\section{Throughput of the DL transmissions}

During the DL time slot $t_{i}^{D}$, the power of the RF signal received by $U_{i}$ is denoted by $P_{r e c v, i}=P_{B S} h_{i}$, where $P_{B S}$ is the transmit power of the BS.

Since only a fraction of the received signal power is exploited by $U_{i}$ for the ID, the achievable DL throughput $R_{i}^{D}$ of $U_{i}$ can be expressed as the following formula by exploiting the classic Shannon's channel capacity equation:

$$
R_{i}^{D}\left(t_{i}^{D}, \rho_{i}\right)=t_{i}^{D} \log _{2}\left[1+\frac{\left(1-\rho_{i}\right) P_{r e c v, i}}{\sigma^{2}}\right],[\mathrm{bit} / \mathrm{Hz}],
$$

The DL throughput of (2) can also be regarded as the bandwidth efficiency of the DL data transfer. Therefore, the bandwidth term of the classic Shannon's channel capacity equation is not included in (2).

\section{Throughput of the UL transmissions}

The total energy harvested by $U_{i}$ is the sum of the energy harvested during the DL time slots set $\left\{t_{j}^{D} \mid j \neq i\right\}$, when $U_{i}$ operates in the $\mathrm{EH}$ mode, and the energy harvested during its dedicated DL time slot $t_{i}^{D}$, when $U_{i}$ operates in both of the EH and ID modes simultaneously. The total energy harvested by $U_{i}$ can then be further expressed as

$$
E_{r e c v, i}\left(\mathbf{t}^{D}, \rho_{i}\right)=\beta_{i} P_{r e c v, i}\left(\sum_{j \neq i} t_{j}^{D}+t_{i}^{D} \rho_{i}\right),
$$

where $\beta_{i}$ represents the efficiency of converting the alternativecurrent (AC) carried by the RF signal to the DC that can drive any electronic load. Here, for simplicity, the energy conversion efficiency $\beta_{i}$ is assumed to be a unity.

Since the energy harvested by $U_{i}$ during the DL transmission is fully exploited for powering its own UL transmission, with the aid of (3), the achievable UL throughput $R_{i}^{U}$ of $U_{i}$ can then be formulated as

$$
\begin{aligned}
& R_{i}^{U}\left(\mathbf{t}^{D}, t_{i}^{U}, \rho_{i}\right) \\
& =t_{i}^{U} \log _{2}\left[1+\frac{h_{i} P_{r e c v, i}\left(\sum_{j \neq i} t_{j}^{D}+t_{i}^{D} \rho_{i}\right)}{t_{i}^{U} \sigma^{2}}\right],[\mathrm{bit} / \mathrm{Hz}],
\end{aligned}
$$

which can also be regarded as the bandwidth efficiency of the UL data transfer.

In our model, the diverse minimum throughput requirements of the UEs' DL transmissions can be represented by the set $\mathbf{D}=\left\{D_{1}, \cdots, D_{K}\right\}$. Our ultimate objective is to maximize the throughput of the UL transmissions subject to the constraint that every UE's achievable DL throughput should satisfy its minimum requirement by jointly optimizing the durations of the time slots in the DL set $\mathbf{t}^{D}$ and those of the time slots in the UL set $\mathbf{t}^{U}$ as well as the signal splitting strategies adopted by the UEs during their dedicated DL time slots. The signal splitting strategies are represented by the PS factors in the set $\boldsymbol{\rho}=\left\{\rho_{i} \mid 1 \leq \rho_{i} \leq K\right\}$. Furthermore, our model focuses on both of the sum-throughput maximisation for achieving the upper-bound of the UEs' UL transmissions and the fair-throughput maximisation for ensuring the UEs' fairness during their UL transmissions.

\section{Sum-ThroughPUT MAXIMIZATION}

In this section, the sum-throughput maximization problem is formulated, and then it is transformed into a convex problem, which can be solved by the classic method of the 
Lagrange multipliers. With the aid of equations (1)-(4), the sum-throughput maximization problem can be formulated as

$$
\begin{aligned}
(\mathrm{P} 1): \max _{\mathbf{t}^{D}, \mathbf{t}^{U}, \boldsymbol{\rho}} & \sum_{i=1}^{K} R_{i}^{U}\left(\mathbf{t}^{D}, t_{i}^{U}, \rho_{i}\right) \\
\text { s.t. } \quad & R_{i}^{D}\left(t_{i}^{D}, \rho_{i}\right) \geq D_{i}, \\
& \sum_{i=1}^{K}\left(t_{i}^{D}+t_{i}^{U}\right) \leq T_{\text {tra }}, \\
& 0 \leq \rho_{i} \leq 1,
\end{aligned}
$$

where $i=1, \cdots, K$ denotes the indices of the UEs. Since $R_{i}^{U}\left(\mathbf{t}^{D}, t_{i}^{U}, \rho_{i}\right)$ of (4) and $R_{i}^{D}\left(t_{i}^{D}, \rho_{i}\right)$ of (2) are neither convex nor concave functions according to the definition of convexity, (P1) is thus a non-convex problem with respect to the variables $\mathbf{t}^{D}, \mathbf{t}^{U}$ and $\boldsymbol{\rho}$. As a result, (P1) has to be equivalently transformed into a convex problem by introducing a new set of variables $\boldsymbol{\mu}=\left\{\mu_{i} \mid i=1, \cdot, K\right\}$ for substituting the original set of variables $\boldsymbol{\rho}=\left\{\rho_{i} \mid i=1, \cdots, K\right\}$. The $i$-th entry $\mu_{i}$ is expressed as

$$
\mu_{i}=t_{i}^{D} \rho_{i}, i=1, \cdots, K
$$

Accordingly, the expression of the achievable DL throughput $R_{i}^{D}$ of $U_{i}$ during its dedicated DL time slot $t_{i}^{D}$ can be reformulated as

$$
R_{i}^{D}\left(t_{i}^{D}, \mu_{i}\right)=t_{i}^{D} \log _{2}\left(1+\gamma_{i}-\gamma_{i} \frac{\mu_{i}}{t_{i}^{D}}\right),
$$

where $\gamma_{i}=\frac{P_{\text {recv }, i}}{\sigma_{2}}$ for all $i=1, \cdots, K$ representing the signal-to-noise-ratio (SNR) of $U_{i}$ during $t_{i}^{D}$. The set of the UEs' SNRs during their DL transmissions is denoted as $\gamma=\left\{\gamma_{i} \mid i=1, \cdots, K\right\}$. The expression of the achievable UL throughput $R_{i}^{U}$ of $U_{i}$ during its assigned UL time slot $t_{i}^{U}$ can be further derived as

$$
R_{i}^{U}\left(\mathbf{t}^{D}, t_{i}^{U}, \mu_{i}\right)=t_{i}^{U} \log _{2}\left(1+\frac{h_{i} \gamma_{i}\left(\sum_{j \neq i} t_{j}^{D}+\mu_{i}\right)}{t_{i}^{U}}\right),
$$

while the PS factor of $U_{i}$ during its assigned DL time slot $t_{i}^{D}$ can be expressed as

$$
\rho_{i}=\frac{\mu_{i}}{t_{i}^{D}} .
$$

Therefore, the original optimisation problem (P1) can be reformulated as

$$
\begin{aligned}
(\mathrm{P} 2): \max _{\mathbf{t}^{D}, \mathbf{t}^{U}, \boldsymbol{\mu}} & \sum_{i=1}^{K} R_{i}^{U}\left(\mathbf{t}^{D}, t_{i}^{U}, \mu_{i}\right) \\
\text { s.t. } \quad & R_{i}^{D}\left(t_{i}^{D}, \mu_{i}\right) \geq D_{i}, \\
& \sum_{i=1}^{K}\left(t_{i}^{D}+t_{i}^{U}\right) \leq T_{\text {tra }}, \\
& 0 \leq \mu_{i} \leq t_{i}^{D},
\end{aligned}
$$

where $i=1, \cdots, K$. Since $f\left(\mathbf{t}^{D}, \mu_{i}\right)=\log _{2}[1+$ $\left.h_{i} \gamma_{i}\left(\sum_{j \neq i} t_{j}^{D}+\mu_{i}\right)\right]$ is a concave function, its log-affine $R_{i}^{U}\left(\mathbf{t}^{D}, t_{i}^{U}, \mu_{i}\right)$ is concave as well. Therefore, the objective function (10) of the alternative optimisation problem (P2), which is the sum of a range of concave functions, can be readily proved to be concave with respect to the variables $\mathbf{t}^{D}$,
$\mathbf{t}^{U}$ and $\boldsymbol{\mu}$. Furthermore, $R_{i}^{D}\left(t_{i}^{D}, \mu_{i}\right)$ in (10a) is also a concave function with these deciding variables since its Hessian matrix is positive semi-definite, while the constrains (10b) and (10c) are both affine. As a result, (P2) is a convex optimization problem.

Observe from the optimisation problem (P2) that the DL transmission requirement $D_{i}$ of $U_{i}$ should be higher than zero and smaller than its maximum achievable DL throughput $R_{i}^{D}$, when $U_{i}$ exploits all its received RF signal for the ID by completely sacrificing its EH function. The Lagrange function of (P2) can be then formulated as

$$
\begin{aligned}
\mathcal{L}\left(\mathbf{t}^{D}, \mathbf{t}^{U}, \boldsymbol{\mu}, \lambda, \boldsymbol{\xi}\right) & =\sum_{i=1}^{K} R_{i}^{U}\left(\mathbf{t}^{D}, t_{i}^{U}, \mu_{i}\right) \\
& +\lambda\left[T_{\text {tra }}-\sum_{i=1}^{K}\left(t_{i}^{D}+t_{i}^{U}\right)\right] \\
& +\sum_{i=1}^{K} \xi_{i}\left[R_{i}^{D}\left(t_{i}^{D}, \mu_{i}\right)-D_{i}\right],
\end{aligned}
$$

where $\lambda$ and $\boldsymbol{\xi}=\left\{\xi_{i} \mid i=1, \cdots, K\right\}$ are the corresponding Lagrangian multipliers. Moreover, the dual function of (P2) can be expressed as

$$
\mathcal{G}(\lambda, \boldsymbol{\xi})=\sup \mathcal{L}\left(\mathbf{t}^{D}, \mathbf{t}^{U}, \boldsymbol{\mu}, \lambda, \boldsymbol{\xi}\right) .
$$

Since (P2) is a convex optimization problem, its optimal solutions, $\left\{\mathbf{t}^{D *}, \mathbf{t}^{U *}, \boldsymbol{\mu}^{*}, \lambda^{*}, \boldsymbol{\xi}^{*}\right\}$, have to satisfy the following Karush-Kuhn-Tucker (KKT) conditions:

$$
\begin{aligned}
& \ln \left(1+y_{i}\right)-\frac{y_{i}}{1+y_{i}}=\lambda \ln 2 \\
& \sum_{j \neq i} \frac{\gamma_{j} h_{j}}{1+y_{j}}+\xi_{i}\left[\ln \left(1+\gamma_{i}-z_{i}\right)+\frac{z_{i}}{1+r_{i}-z_{i}}\right]=\lambda \ln 2 \\
& \frac{\gamma_{i} h_{i}}{1+y_{i}}=\xi_{i} \frac{\gamma_{i}}{1+\gamma_{i}-z_{i}} \\
& \lambda\left[T_{\text {tra }}-\sum_{i=1}^{K}\left(t_{i}^{U}+t_{i}^{D}\right)\right]=0 \\
& \xi_{i}\left[t_{i}^{D} \log _{2}\left(1+\gamma_{i}-z_{i}\right)-D_{i}\right]=0
\end{aligned}
$$

where we introduce a couple of new variables sets, denoted by $\mathbf{y}=\left\{y_{i} \mid i=1, \cdots, K\right\}$ and $\mathbf{z}=\left\{z_{i} \mid i=1, \cdots, K\right\}$. Their $i$-th entries can be expressed as

$$
\begin{aligned}
y_{i} & =h_{i} \gamma_{i} \frac{\sum_{j \neq i} t_{j}^{D}+\mu_{i}}{t_{i}^{U}}, \\
z_{i} & =\gamma_{i} \frac{\mu_{i}}{t_{i}^{D}}
\end{aligned}
$$

respectively, for $i=1, \cdots, K$. According to (14) and (15), we can find that $\lambda \neq 0$ and $\xi_{i} \neq 0$.

Given a specific value of the Lagrange multiplier $\lambda$ and according to (13)-(19), the resultant optimal value $t_{i}^{D *}$ of the duration of the $U_{i}$ 's assigned DL time slot can be derived as

$$
t_{i}^{D *}=\frac{D_{i}}{\log _{2}\left(1+\gamma_{i}-z_{i}^{*}\right)} .
$$


Furthermore, the optimal value $t_{i}^{U *}$ of the duration of the $U_{i}$ 's assigned UL time slot can be obtained as

$$
t_{i}^{U *}=h_{i} \gamma_{i} \frac{\sum_{j \neq i} t_{j}^{D *}+\mu_{i}^{*}}{y_{i}^{*}}, .
$$

The optimal value of the intermediate variable $\mu_{i}^{*}$ is formulated as

$$
\mu_{i}^{*}=\frac{z_{i}^{*} t_{i}^{D *}}{\gamma_{i}} .
$$

In equations (20), (21) and (22), $y_{i}^{*}$ and $z_{i}^{*}$ are the solutions to the following equations:

$$
\begin{aligned}
\ln \left(1+y_{i}\right)-\frac{y_{i}}{1+y_{i}} & =\lambda \ln 2 \\
h_{i}\left(1+\gamma_{i}-z_{i}\right) \ln (1 & \left.+\gamma_{i}-z_{i}\right)+h_{i} z_{i} \\
& =\left(1+y_{i}\right) \lambda \ln 2-\sum_{j \neq i} \gamma_{j} h_{j} .
\end{aligned}
$$

The expression on the left side of (23) increases monotonically with respect to the variable $y_{i}$, while the expression on the left side of equation (24) decreases monotonically with respect to the variable $z_{i}$. As a result, $y_{i}^{*}$ can be calculated first by invoking the classic bisection method. Substituting $y_{i}^{*}$ into (24), $z_{i}^{*}$ can also be calculated by invoking the classic bisection method.

Given the specific value of $\lambda$, we have obtained the optimal values of $\mathbf{t}^{U *}, \mathbf{t}^{D *}, \boldsymbol{\mu}^{*}$ and $\boldsymbol{\xi}^{*}$, which satisfy the equalities of (13)-(15) and (17). Then, the sub-gradient descent is invoked for iteratively obtaining the optimal Lagrange multiplier $\lambda^{*}$. The sub-gradient of $\mathcal{G}(\lambda, \boldsymbol{\xi})$ with respect to the Lagrange multiplier $\lambda$, which is denoted by $p(\lambda)$, can be further expressed as

$$
p(\lambda)=T_{\text {tra }}-\sum_{i=1}^{K}\left(t_{i}^{D}+t_{i}^{U}\right) .
$$

With the aid of (25), we can iteratively obtain the optimal Lagrange multiplier $\lambda^{*}$. We update $\lambda$ by the formula $\lambda^{(n)}=$ $\lambda^{(n-1)}-p\left(\lambda^{(n-1)}\right) \triangle_{\lambda}$ in each iteration, where $n$ denotes the $n$-th iteration and $\Delta_{\lambda}$ represents the step length of each iteration. Substituting $\lambda^{(n)}$ into (20)-(24), we may obtain the corresponding values of $t_{i}^{D}$ and $t_{i}^{U}$ and hence derive the specific value of $p\left(\lambda^{(n)}\right)$. The iteration continues until we find the optimal $\lambda^{*}$, which makes $\left|p\left(\lambda^{*}\right)\right|$ smaller than the specific error tolerance $\delta$. Finally, the PS factors set $\rho^{*}$ can be calculated by invoking (9). The procedure for iteratively solving the alternative optimisation problem (P2) is detailed in the pseudo code of Algorithm 1.

\section{FAIR-THROUGHPUT MAXIMIZATION}

In order to achieve a better sum-throughput, more resources are inclined to be allocated to the UEs having better channel qualities between the BS. Since the channel qualities are largely determined by the large-scale channel attenuation, such as the path-loss, the UEs close to the BS may gain more resources for harvesting energy from the BS's DL transmission and for sending their own data to the BS during their UL transmissions. As a result, the UEs relatively far away from the BS may not be allocated sufficient resources for their

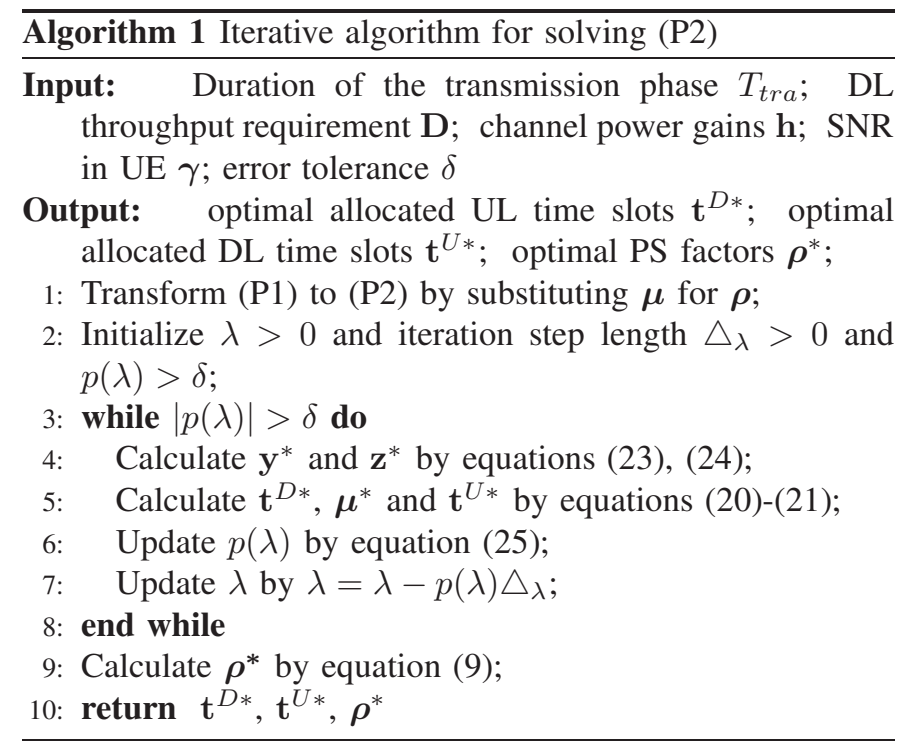

own operations. This is regarded as the classic near-far effect, which yields the fairness issue among the UEs in the DEIN.

In order to overcome the classic near-far effect during the resource allocation, ensuring the fairness among the UEs' UL transmissions becomes our prim objective, which yields the maximization of the so-called fair-throughput. Since fairthroughput represents the minimum throughput among all the UEs during their UL transmissions, we impose a constraint on the throughput of the UEs' UL transmissions, which is expressed as $R_{i}^{U}\left(\mathbf{t}^{D}, t_{i}^{U}, \rho_{i}\right) \geq R$, for $i=1, \cdots, K$, where $R$ represents the so-called fair-throughput. According to the system model of Section II, the fair-throughput maximization problem (P3) can be formulated as

$$
\begin{aligned}
(\mathrm{P} 3): \max _{\mathbf{t}^{D}, \mathbf{t}^{U}, \boldsymbol{\mu}} & R \\
\text { s.t. } & R_{i}^{D}\left(t_{i}^{D}, \mu_{i}\right) \geq D_{i}, \\
& R_{i}^{U}\left(\mathbf{t}^{D}, t_{i}^{U}, \mu_{i}\right) \geq R, \\
& 0 \leq \mu_{i} \leq t_{i}^{D}, \\
& \sum_{i=1}^{K}\left(t_{i}^{D}+t_{i}^{U}\right) \leq T_{\text {tra }},
\end{aligned}
$$

where $\mu_{i}=t_{i}^{D} \rho_{i}$ for all $i=1, \cdots, K$ is adopted for ensuring the concavity of both the achievable DL throughput $R_{i}^{D}\left(t_{i}^{D}, \mu_{i}\right)$ of $U_{i}$ during its DL time slot $t_{i}^{D}$ and the achievable UL throughput $R_{i}^{U}\left(\mathbf{t}^{\mathbf{D}}, t_{i}^{U}, \mu_{i}\right)$ during its UL time slot $t_{i}^{U}$, which have been proved in Section III. As a result, the fair-throughput maximisation problem (P3) can be readily proved to be a convex optimization problem. Note that the achievable DL throughput $R_{i}^{D}\left(t_{i}^{D}, \mu_{i}\right)$ is an increasing function with respect to $t_{i}^{D}$, while the achievable UL throughput $R_{i}^{U}\left(\mathbf{t}^{D}, t_{i}^{U}, \mu_{i}\right)$ is also an increasing function with respect to $t_{i}^{D}$ and $t_{i}^{U}$. Therefore, the fair-throughput $R$ increases when $t=\sum_{i=1}^{K} t_{i}^{D}+t_{i}^{U}$ increases. As a result, we may iteratively solve the following convex optimisation problem (P4) in order 
to maximize the fair-throughput $R$ :

$$
\begin{aligned}
(\mathrm{P} 4): \min _{\mathbf{t}^{D}, \mathbf{t}^{U}, \boldsymbol{\mu}} & \sum_{i=1}^{K}\left(t_{i}^{D}+t_{i}^{U}\right) \\
\text { s.t. } & R_{i}^{D}\left(t_{i}^{D}, \mu_{i}\right) \geq D_{i}, \\
& R_{i}^{U}\left(\mathbf{t}^{D}, t_{i}^{U}, \mu_{i}\right) \geq R, \\
& 0 \leq \mu_{i} \leq t_{i}^{D},
\end{aligned}
$$

where $i=1, \cdots, K$. The Lagrange function of (P4) is further expressed as

$$
\begin{aligned}
\mathcal{L}\left(\mathbf{t}^{D}, \mathbf{t}^{U}, \boldsymbol{\mu}, \boldsymbol{\lambda}, \boldsymbol{\xi}\right) & =\sum_{i=1}^{K}\left(t_{i}^{D}+t_{i}^{U}\right) \\
& +\sum_{i=1}^{K} \xi_{i}\left[D_{i}-R_{i}^{D}\left(t_{i}^{D}, \mu_{i}\right)\right] \\
& +\sum_{i=1}^{K} \lambda_{i}\left[R-R_{i}^{U}\left(\mathbf{t}^{D}, t_{i}^{U}, \mu_{i}\right)\right],
\end{aligned}
$$

where $\boldsymbol{\lambda}=\left\{\lambda_{i} \mid i=1, \cdots, K\right\}$ and $\boldsymbol{\xi}=\left\{\xi_{i} \mid i=1, \cdots, K\right\}$ are the corresponding Lagrangian multipliers. The dual function of (P4) then can be expressed as

$$
\mathcal{G}(\boldsymbol{\lambda}, \boldsymbol{\xi})=\inf \mathcal{L}\left(\mathbf{t}^{D}, \mathbf{t}^{U}, \boldsymbol{\mu}, \boldsymbol{\lambda}, \boldsymbol{\xi}\right) .
$$

Similar to the method invoked for solving the sumthroughput maximisation problem (P2), the KKT conditions are also exploited for solving the fair-throughput maximisation problem (P4). Hence, given a range of specific values for the multiplier set $\boldsymbol{\lambda}=\left\{\lambda_{i} \mid i=1, \cdots, K\right\}$, the optimal value of the duration of the DL time slot $t_{i}^{D *}$, that of the duration of the UL time slot $t_{i}^{U *}$ and that of the intermediate variable $\mu_{i}^{*}$ can still be expressed by (20)-(22). Furthermore, $y_{i}^{*}$ and $z_{i}^{*}$ can be obtained by solving the following equations:

$$
\begin{aligned}
& \ln \left(1+y_{i}\right)-\frac{y_{i}}{1+y_{i}}=\frac{\ln 2}{\lambda_{i}}, \\
& \left(1+\gamma_{i}-z_{i}\right) \ln \left(1+\gamma_{i}-z_{i}\right)+z_{i} \\
& =\frac{1+y_{i}}{\lambda_{i} h_{i}} \ln 2-\frac{\sum_{j \neq i} \lambda_{j} \gamma_{j} h_{j}}{\lambda_{i} h_{i}} .
\end{aligned}
$$

Relying on the monotonous properties of the expressions on the left side of the equalities of (30) and (31), we can readily obtain the solutions of $y_{i}^{*}$ and $z_{i}^{*}$ by invoking the classic bisection method. The sub-gradient of $\mathcal{G}(\boldsymbol{\lambda}, \boldsymbol{\xi})$ with respect to $\boldsymbol{\lambda}$, which is denoted by $p(\boldsymbol{\lambda})=\left\{p\left(\lambda_{i}\right) \mid i=1, \cdots, K\right\}$, can be further expressed as

$$
p\left(\lambda_{i}\right)=t_{i}^{U *} \log _{2}\left(1+y_{i}^{*}\right)-R,
$$

for all $i=1, \cdots, K$. We iteratively update the Lagrange multiplier set $\boldsymbol{\lambda}$ by $\boldsymbol{\lambda}^{(n)}=\boldsymbol{\lambda}^{(n-1)}-p(\boldsymbol{\lambda}) \Delta_{\boldsymbol{\lambda}}$ in each iteration, where $n$ denotes the $n$-th iteration and $\Delta_{\boldsymbol{\lambda}}$ represents the step length of the iteration. The iteration for obtaining the optimal Lagrange multiplier set $\lambda^{*}$ terminates until the subgradient of the dual function $\mathcal{G}(\boldsymbol{\lambda}, \boldsymbol{\xi})$ satisfies the condition of $\left|p\left(\boldsymbol{\lambda}^{*}\right)\right| \leq \delta_{\boldsymbol{\lambda}}$, where $\delta_{\boldsymbol{\lambda}}$ represents the absolute error tolerance of the Lagrange multiplier set $\lambda$.

We reduce the fair-throughput $R$ after obtaining the optimal result $t^{*}$ by solving the alternative optimisation problem (P4),

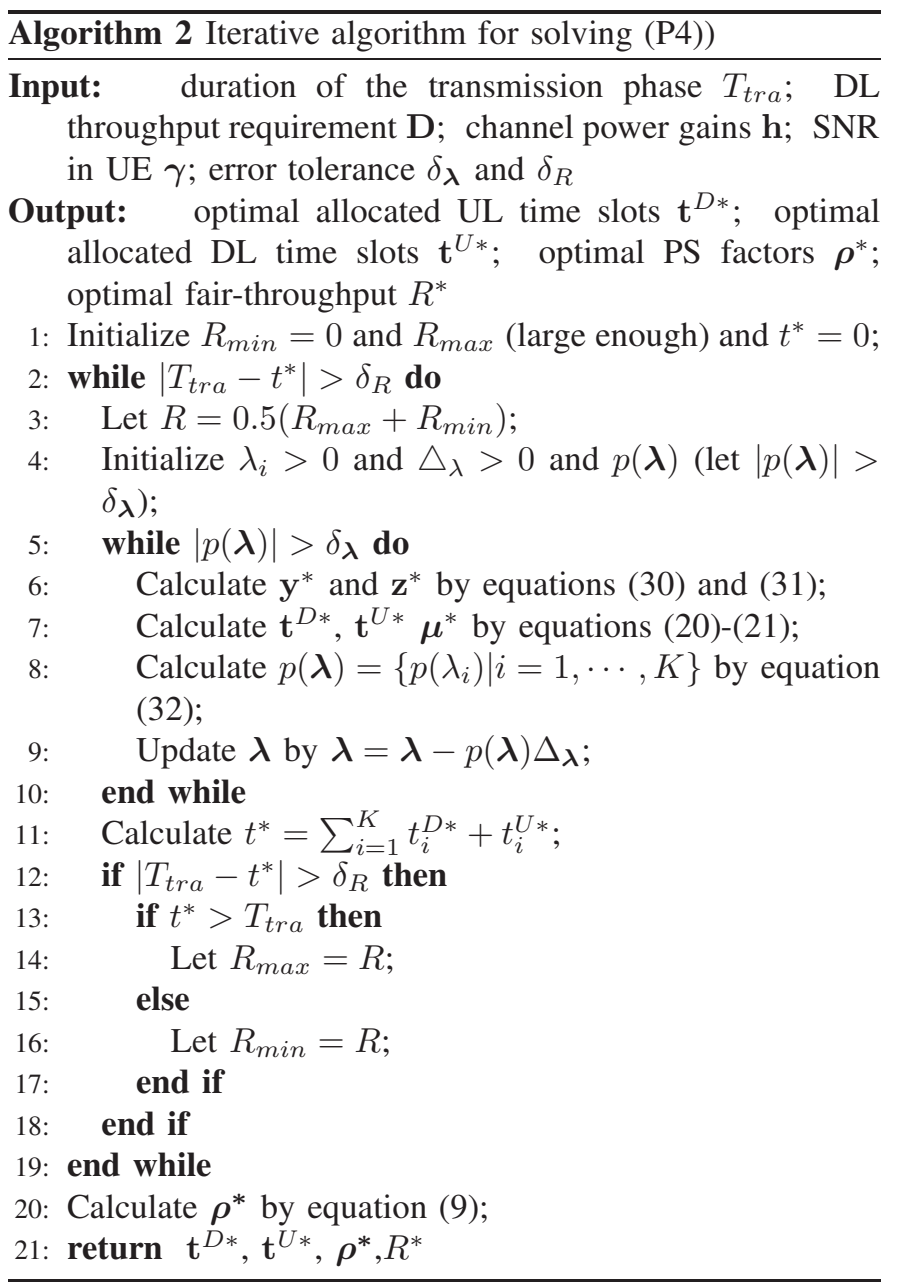

if the optimal result $t^{*}$ is higher than the duration $T_{\text {tra }}$ of the transmission phase, say $t^{*}>T_{t r a}$. By contrast, if the optimal result $t^{*}$ is lower than the duration $T_{t r a}$ of the transmission phase, say $t^{*}<T_{\text {tra }}$, we have to increase the fairthroughput $R$. This iteration process terminates until we have $\left|T_{\text {tra }}-t^{*}\right|<\delta_{R}$, which yields the maximum fair-throughput $R^{*}$. Here, $\delta_{R}$ represents the error tolerance. The iterative algorithm of solving the alternative optimisation problem (P4) is tailored in Algorithm 2.

\section{Numerical RESUlT}

In this section, the numerical results of the maximum sumthroughput obtained by solving the optimisation problem (P1) and those of the maximum fair-throughput obtained by solving the optimisation problem (P3) are compared with each other in a typical DEIN consisting of a BS and several UEs. Without loss of generality, the Additive-White-Gaussian-Noise (AWGN) channel as well as the path loss are conceived. Therefore, the DL and UL channel power gains are modelled by $h_{i}=g_{i}=10^{-3} Y_{i}^{-\alpha}$, for all $i=1, \cdots, K$, where $Y_{i}$ represents the distance between the BS and $U_{i}$. The exponent is set to be $\alpha=2$ for representing the short-range free-space path loss model. A $30 \mathrm{~dB}$ signal power attenuation in average is assumed at a reference distance of $1 \mathrm{~m}$ for this channel model. The noise power of the information decoder is set to be $-50 \mathrm{dBm}$, while the channel noise is ignored. 


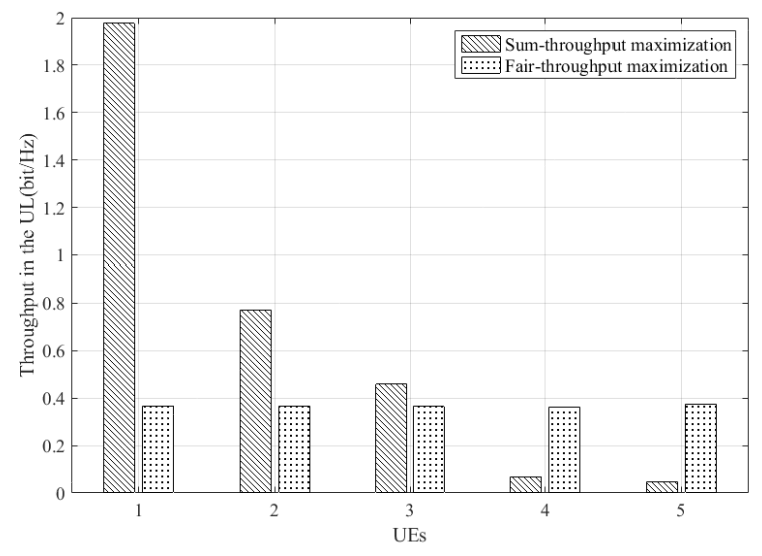

Fig. 3: The individual throughput of the UEs' UL transmission.

We first compare the UE's individual UL throughput obtained by solving the sum-throughput maximization problem (P1) to that obtained by solving the fair-throughput maximization problem (P4). The transmit power $P_{B S}$ is set to be 30 $\mathrm{dBm}$. We have $K=5$ UEs in total in the DEIN. The distances from the UEs to the BS are $\left\{Y_{1}=4, Y_{2}=5, Y_{3}=5.5, Y_{4}=\right.$ $\left.9, Y_{5}=10\right\} \mathrm{m}$, while the minimum requirements of the UEs' DL throughput are $\left\{D_{1}=0.5, D_{2}=0.4, D_{3}=0.8, D_{4}=\right.$ $\left.0.3, D_{5}=0.2\right\} \mathrm{bit} / \mathrm{Hz}$. The duration of the transmission phase is $T_{\text {tra }}=1 \mathrm{~s}$.

As illustrated in Fig.3, the UEs within the proximity of the BS, such as $U_{1}$ and $U_{2}$, are capable of transferring more data during their UL transmissions than the UEs far away from the BS, such as $U_{4}$ and $U_{5}$, if we aim for maximizing the sumthroughput of the UEs' UL transmission. In order to achieve this objective, more time is assigned to the UEs having better channel qualities, which results in the substantial unfairness among the UEs. As a result, in order to attain a fair resource allocation scheme, the maximization of the fair-throughput is studied in order to ensure the fairness among the UEs by suffering somewhat degradation of the sum-throughput. We can observe from Fig.3 that in order to maximize the fairthroughput, the actual UL throughput of different UEs are soundly fair by distributing more time to UEs having worse channel qualities for the sake of overcoming the adverse nearfar effect.

Furthermore, we plot the PS strategy for these five UEs during their DL transmissions in Fig.4. We first focus on the sum-throughput maximisation. Since $U_{1}$ and $U_{2}$ are very close to the BS, they only exploit a very small fraction of their received signal for the information decoding so as to satisfy their DL throughput requirement. The rest of their received signal is all converted to the energy, which is exploited for supporting their UL transmissions. As a result, $U_{1}$ and $U_{2}$ are capable of achieving higher UL transmission throughput. By contrast, since $U_{4}$ and $U_{5}$ are far away from the BS, they have to exploit all their received signals for the information decoding. As a result, they do not harvest sufficient energy for supporting their UL transmissions. Hence, they suffer from very low UL transmission throughput. Note that when the fair-

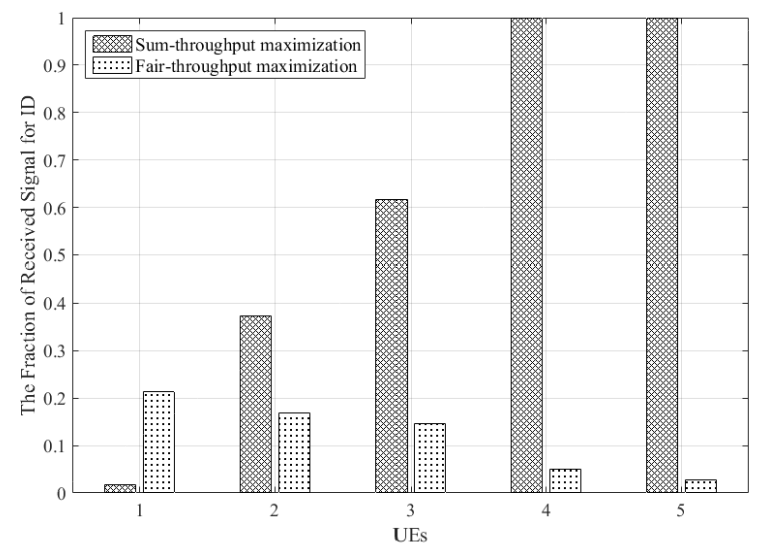

Fig. 4: The PS strategies of the UEs during their DL transmissions.

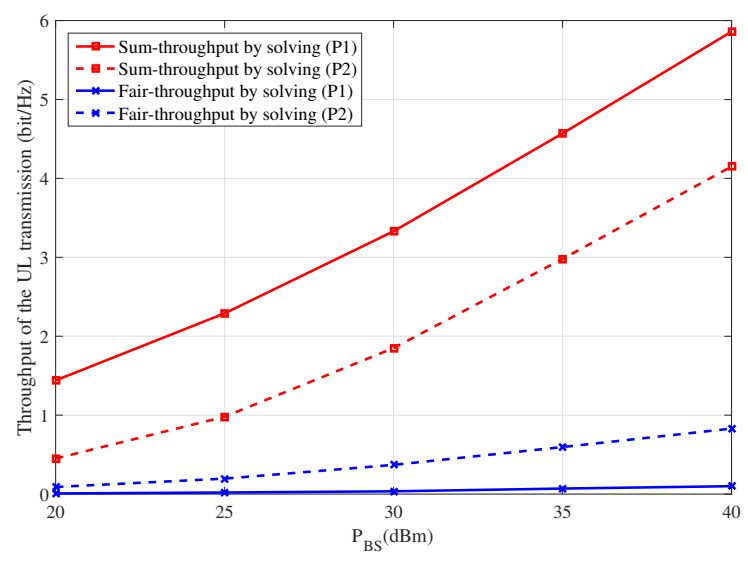

Fig. 5: Throughput of the UL transmission versus the transmit power of the BS.

throughput maximisation is invoked in our resource allocation and strategy selection schemes, all the UEs choose moderate PS strategies in order to achieve the fairness of their UL transmissions.

We further plot both of the sum-throughput and the fairthroughput against the transmit power $P_{B S}$ of the $\mathrm{BS}$ in Fig.5, where we adopt the same parameter setting as those for obtaining the numerical results of Fig.3. Observe from Fig.5 that when the transmit power $P_{B S}$ of the BS increases, both of the sum-throughput obtained by solving the optimisation problem (P1) and the fair-throughput obtained by solving the optimisation problem (P4) increase. Furthermore, the sumthroughput is more sensitive to the increase of $P_{B S}$ than the fair-throughput. Without considering the fairness among the UEs, the UL throughputs of the UEs near the BS may be significantly increased by increasing the transmit power of $P_{B S}$. However, the UL throughputs of the UEs far away from the BS may be improved little due to the signal propagation of long distances. Hence, the substantial increase of the sumthroughput is mainly contributed by the UEs near the BS. Since the fair-throughput mainly depends on the UEs having 


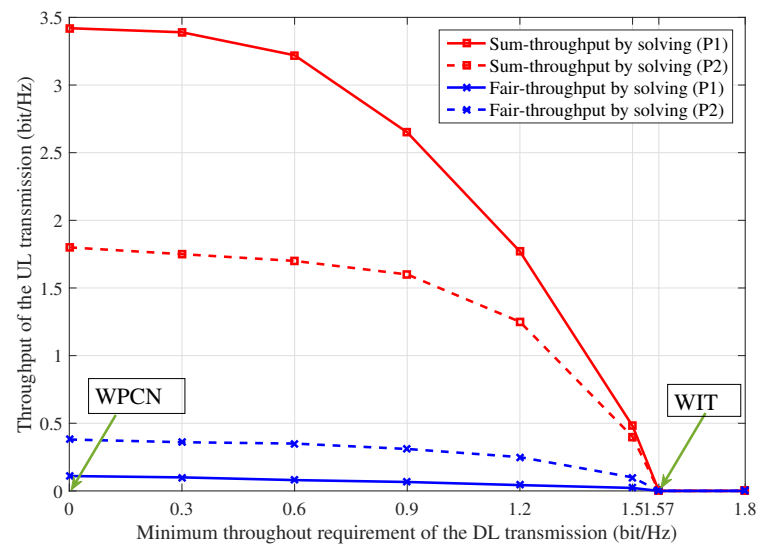

Fig. 6: Throughput of the UL transmission versus the minimum throughput requirement in the DL transmission.

worse channel qualities, it may not be improved a lot by increasing the transmit power $P_{B S}$ of the BS.

We plot both of the sum-throughput and the fair-throughput of the UL transmission against the minimum throughput requirement of the DL transmission in Fig.6. For simplicity, we set the minimum DL throughput requirement identical for all the UEs. Note that if the minimum DL throughput requirement falls to zero, our DEIN becomes a typical WPCN. In the WPCN, the DL transmission of the BS does not carry any requested data. The DL signal is only for transferring the energy to the UEs. Observe from Fig.6 that as we increase the minimum DL throughput requirement, both of the sumthroughput and fair-throughput of the UL transmission are reduced. We also have an interesting observation that when the minimum DL throughput requirement is lower than 0.3 $\mathrm{bit} / \mathrm{Hz}$, it has little influence on both of the sum-throughput and the fair-throughput of the UL transmission. The observation indicates that our DEIN can efficiently support the low-rate DL transmission, such as the signalling exchange, while fulfilling the wireless charging tasks, without any significant loss of the UEs' UL transmissions. As the minimum throughput requirement of the DL transmission continually increases, both of the sum-throughput and the fair-throughput gradually become zero. This is because a large portion of the received RF signal of the DL transmission is fully exploited for the information decoder in order to satisfy the harsh DL throughput requirement and hence the UEs cannot harvest sufficient energy for supporting their own UL transmissions. As shown in Fig.6, the UL throughput reduces to zero, when the minimum DL throughput requirement increases to $1.57(\mathrm{bit} / \mathrm{Hz})$. At this moment, the UEs completely sacrifice the function of the energy harvesting in order to achieve the minimum DL throughput requirements, which makes our DEIN model a conventional wireless information transfer (WIT) system. If the DL throughput requirement is higher than $1.57(\mathrm{bit} / \mathrm{Hz})$, this requirement is beyond the transmission capability of the DEIN in the current parameter settings.

\section{CONCLUSION}

This paper has studied a novel DEIN model, where the BS simultaneously transmit the data and energy during the DL transmissions and the UEs harvest the energy from the DL signals for powering their own UL transmissions. In order to avoid any collision and interference, a TDMA protocol is adopted in the MAC layer for both the DL and UL transmissions. At a UE's end, the received RF signal is split in the power domain. One portion of the signal is for the information decoding, while the other is for the energy harvesting. Relying on the classic convex optimization theory, both of the sum-throughput and the fair-throughput are maximised by optimizing both of the time slots allocation and the PS factors. Iterative algorithms are proposed for numerically solving the throughput maximization problems. Furthermore, our numerical results demonstrate the advantage of our DEIN over the WPCN and the WIT systems.

\section{REFERENCES}

[1] W. Ejaz, M. Naeem, A. Shahid, A. Anpalagan, and M. Jo, "Efficient energy management for the internet of things in smart cities," IEEE Communications Magazine, vol. 55, no. 1, pp. 84-91, January 2017.

[2] F. Zhu, Z. Li, S. Chen, and G. Xiong, "Parallel transportation management and control system and its applications in building smart cities," IEEE Transactions on Intelligent Transportation Systems, vol. 17, no. 6, pp. 1576-1585, June 2016.

[3] A. Boustani, A. Maiti, S. Y. Jazi, M. Jadliwala, and V. Namboodiri, "Seer grid: Privacy and utility implications of two-level load prediction in smart grids," IEEE Transactions on Parallel and Distributed Systems, vol. 28, no. 2, pp. 546-557, February 2017.

[4] X. Chen, L. Wang, J. Ding, and N. Thomas, "Patient flow scheduling and capacity planning in a smart hospital environment," IEEE Access, vol. 4, pp. 135-148, December 2016.

[5] P. Xu, H. Mei, L. Ren, and W. Chen, "Vidx: Visual diagnostics of assembly line performance in smart factories," IEEE Transactions on Visualization and Computer Graphics, vol. 23, no. 1, pp. 291-300, January 2017.

[6] M. Hassanalieragh, T. Soyata, A. Nadeau, and G. Sharma, "Ur-solarcap: An open source intelligent auto-wakeup solar energy harvesting system for supercapacitor-based energy buffering," IEEE Access, vol. 4, pp. 542-557, January 2016.

[7] D. Porcarelli, D. Spenza, D. Brunelli, A. Cammarano, C. Petrioli, and L. Benini, "Adaptive rectifier driven by power intake predictors for wind energy harvesting sensor networks," IEEE Journal of Emerging and Selected Topics in Power Electronics, vol. 3, no. 2, pp. 471-482, June 2015.

[8] S. Ulukus, A. Yener, E. Erkip, O. Simeone, M. Zorzi, P. Grover, and K. Huang, "Energy harvesting wireless communications: A review of recent advances," IEEE Journal on Selected Areas in Communications, vol. 33, no. 3, pp. 360-381, March 2015.

[9] A. M. Z. L. M. A. S. Prabaharan and K. P. Seng, "Radio frequency energy harvesting and management for wireless sensor net-works," Green Mobile Devices Netw.: Energy Opt. Scav. Tech. CRC Press, pp. 341-368, 2012.

[10] L. R. Varshney, "Transporting information and energy simultaneously," in 2008 IEEE International Symposium on Information Theory, July 2008, pp. 1612-1616.

[11] B. Koo and D. Park, "Interference alignment and wireless energy transfer via antenna selection," IEEE Communications Letters, vol. 18, no. 4, pp. 548-551, April 2014.

[12] L. Liu, R. Zhang, and K. C. Chua, "Wireless information and power transfer: A dynamic power splitting approach," IEEE Transactions on Communications, vol. 61, no. 9, pp. 3990-4001, September 2013.

[13] Y. Dong, M. J. Hossain, and J. Cheng, "Joint power control and time switching for swipt systems with heterogeneous qos requirements," IEEE Communications Letters, vol. 20, no. 2, pp. 328-331, February 2016.

[14] P. Grover and A. Sahai, "Shannon meets tesla: Wireless information and power transfer," in 2010 IEEE International Symposium on Information Theory, June 2010, pp. 2363-2367. 
[15] K. Huang and E. Larsson, "Simultaneous information and power transfer for broadband wireless systems," IEEE Transactions on Signal Processing, vol. 61, no. 23, pp. 5972-5986, Dec 2013.

[16] X. Zhou, R. Zhang, and C. K. Ho, "Wireless information and power transfer in multiuser ofdm systems," in 2013 IEEE Global Communications Conference (GLOBECOM), Dec 2013, pp. 4092-4097.

[17] R. Zhang and C. K. Ho, "Mimo broadcasting for simultaneous wireless information and power transfer," IEEE Transactions on Wireless Communications, vol. 12, no. 5, pp. 1989-2001, May 2013.

[18] H. Ju and R. Zhang, "Throughput maximization in wireless powered communication networks," in 2013 IEEE Global Communications Conference (GLOBECOM), Dec 2013, pp. 4086-4091.

[19] Y. L. Che, L. Duan, and R. Zhang, "Spatial throughput maximization of wireless powered communication networks," IEEE Journal on Selected Areas in Communications, vol. 33, no. 8, pp. 1534-1548, Aug 2015.

[20] H. Ju and R. Zhang, "Optimal resource allocation in full-duplex wirelesspowered communication network," IEEE Transactions on Communications, vol. 62, no. 10, pp. 3528-3540, Oct 2014.

[21] S. Bi, Y. Zeng, and R. Zhang, "Wireless powered communication networks: An overview," IEEE Wireless Communications, vol. 23, no. 2, pp. 10-18, apr 2016. [Online]. Available: http://ieeexplore.iee.org/document/7462480/

[22] O. Ozel, K. Shahzad, and S. Ulukus, "Optimal energy allocation for energy harvesting transmitters with hybrid energy storage and processing cost," IEEE Transactions on Signal Processing, vol. 62, no. 12, pp. 3232-3245, June 2014.

[23] J. Park and B. Clerckx, "Joint wireless information and energy transfer with reduced feedback in mimo interference channels," IEEE Journal on Selected Areas in Communications, vol. 33, no. 8, pp. 1563-1577, August 2015.

[24] W. Sun, E. G. Strm, F. Brnnstrm, and M. R. Gholami, "Random broadcast based distributed consensus clock synchronization for mobile networks," IEEE Transactions on Wireless Communications, vol. 14, no. 6, pp. 3378-3389, June 2015.

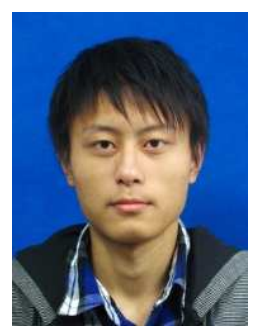

Kesi $\mathbf{L v}$ received his B.S degree from University of Electronic Science and Technology of China (UESTC), China, in 2016. He is now studying for a master's degree at UESTC. His research interests include data and energy integrated communication networks and convex optimization.

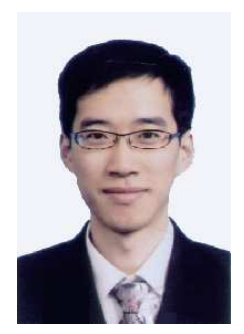

Jie Hu (S'11-M'16) received his B.Eng. and M.Sc. degrees from Beijing University of Posts and Telecommunications, China, in 2008 and 2011, respectively, and received the Ph.D. degree from the Faculty of Physical Sciences and Engineering, University of Southampton, U.K., in 2015. Since March 2016, he has been working with the School of Communication and Information Engineering, University of Electronic Science and Technology of China (UESTC), China, as a Lecturer. His research now is funded by National Natural Science Foundation of China (NSFC). He is also in great partnership with industry, such as Huawei and ZTE. He has acted as a member of technical program committee for several prestigious international conferences, including Globecom 2016/2017, ICC 2017. He has a broad range of interests in wireless communication and networking, such as cognitive radio and cognitive networks, mobile social networks, data and energy integrated communication networks as well as communication and computation convergence.

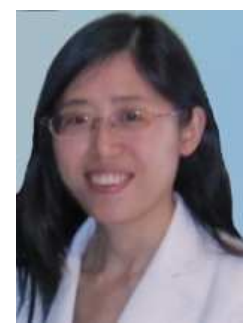

Qin Yu received the B.S. degree in communication engineering from the Chongqing University of Posts and Telecommunications in 1996, and the M.S. and $\mathrm{Ph} . \mathrm{D}$. degrees in communication and information engineering from University of Electronic Science and Technology of China (UESTC) in 2002 and 2006, respectively. She joined the School of Communication and Information Engineering of UESTC in 2007. From 2007 to 2009, she conducted postdoctoral research with professor Zhiguang Qin in information security at UESTC. Her current research interests include wireless networks and information security.

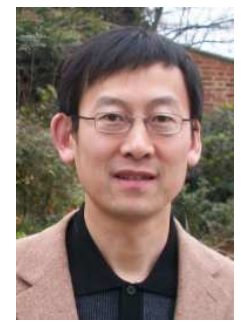

Kun Yang (M'00, SM'07) received his $\mathrm{PhD}$ from the Department of Electronic and Electrical Engineering of University College London (UCL), UK, and MSc and BSc from the Computer Science Department of Jilin University, China. He is currently a Chair Professor in the School of Computer Science \& Electronic Engineering, University of Essex, leading the Network Convergence Laboratory (NCL), UK. He is also an affiliated professor at UESTC, China. Before joining in University of Essex at 2003, he worked at UCL on several European Union (EU) research projects for several years. His main research interests include wireless networks, future Internet technology and network virtualization, mobile cloud computing and networking. He manages research projects funded by various sources such as UK EPSRC, EU FP7/H2020 and industries. He has published $100+$ journal papers. He serves on the editorial boards of both IEEE and nonIEEE journals. He is a Senior Member of IEEE (since 2007) and a Fellow of IET (since 2009). 